\title{
Correction to: The International Mouse Phenotyping Consortium (IMPC): a functional catalogue of the mammalian genome that informs conservation
}

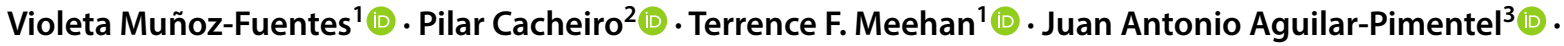 \\ Arthur L. Beaudet ${ }^{15}$. Steve D. M. Brown ${ }^{4}$. Mary E. Dickinson ${ }^{15,17}$. Ann M. Flenniken ${ }^{5,6}$. Paul Flicek ${ }^{1}$. \\ Antonella Galli ${ }^{7}$. Hamed Haseli Mashhadi ${ }^{1}$. Jason D. Heaney ${ }^{15}$. Martin Hrabě de Angelis ${ }^{3,8,9}$. Jong Kyoung Kim ${ }^{10}$. \\ K. C. Kent Lloyd ${ }^{11} \cdot$ Colin McKerlie $^{5,6,12} \cdot$ Hugh Morgan $^{4} \cdot$ Stephen A. Murray ${ }^{13} \cdot$ Lauryl M. J. Nutter $^{5,12}$. \\ Patrick T. Reilly ${ }^{14}$. John R. Seavitt ${ }^{15}$. Je Kyung Seong ${ }^{16} \cdot$ Michelle Simon $^{4} \cdot$ Hannah Wardle-Jones $^{7}$. \\ Ann-Marie Mallon ${ }^{4} \cdot$ Damian Smedley $^{2} \cdot$ Helen E. Parkinson ${ }^{1} \cdot$ the IMPC consortium
}

Published online: 25 January 2019

(c) The Author(s) 2019

\section{Correction to: Conservation Genetics (2018) 19:995-1005 https://doi.org/10.1007/s10592-018-1072-9}

The original publication of this article unfortunately contained the following mistakes.

1. This special issue article had been mistakenly included and published in Volume 19, issue 4, though it should

Violeta Muñoz-Fuentes and Pilar Cacheiro shared first authorship.

The original article can be found online at https://doi.org/10.1007/ s10592-018-1072-9.

Violeta Muñoz-Fuentes

vmunoz@ebi.ac.uk

1 European Molecular Biology Laboratory, European Bioinformatics Institute (EMBL-EBI), Wellcome Genome Campus, Hinxton, Cambridge CB10 1SD, UK

2 Clinical Pharmacology, William Harvey Research Institute, School of Medicine and Dentistry, Queen Mary University of London, Charterhouse Square, London EC1M 6BQ, UK

3 German Mouse Clinic, Institute of Experimental Genetics, Helmholtz Zentrum München, German Research Center for Environmental Health, Ingolstädter Landstrasse 1, 85764 Neuherberg, Germany

4 Medical Research Council Harwell Institute (Mammalian Genetics Unit and Mary Lyon Centre), Harwell, Oxfordshire OX11 0RD, UK

5 The Centre for Phenogenomics, Toronto, ON M5T 3H7, Canada

6 Mount Sinai Hospital, Toronto, ON M5G 1X5, Canada

7 Wellcome Trust Sanger Institute, Cambridge CB10 1SA, UK have been published in the special issue titled, "Conservation of Adaptive Potential". The Publisher regrets the mistake.

2. In the original publication, the article category should read: "Perspective Article".

3. The authorship was incorrect and the correct one is given in this Correction.

8 German Center for Diabetes Research (DZD), Ingolstädter Landstr. 1, 85764 Neuherberg, Germany

9 School of Life Science Weihenstephan, Technische Universität München, Alte Akademie 8, 85354 Freising, Germany

10 Department of New Biology, DGIST, Daegu 42988, Republic of Korea

11 Mouse Biology Program, University of California, Davis, CA 95618, USA

12 The Hospital for Sick Children, Toronto, ON M5G 1X84, Canada

13 The Jackson Laboratory, Bar Harbor, ME 04609, USA

14 PHENOMIN-iCS, 1 Rue Laurent Fries, 67404 Illkirch Cedex, Alsace, France

15 Department of Molecular and Human Genetics, Baylor College of Medicine, Houston, TX 77030, USA

16 Laboratory of Developmental Biology and Genomics, College of Veterinary Medicine, Interdisciplinary Program for Bioinformatics and Program for Cancer Biology, Seoul National University, Seoul, Republic of Korea

17 Department of Molecular Physiology and Biophysics, Baylor College of Medicine, Houston, TX 77030, USA 
Open Access This article is distributed under the terms of the Creative Commons Attribution 4.0 International License (http://creat ivecommons.org/licenses/by/4.0/), which permits unrestricted use, distribution, and reproduction in any medium, provided you give appropriate credit to the original author(s) and the source, provide a link to the Creative Commons license, and indicate if changes were made. 